\title{
Traduire la créolité métisse dans les Pays tchèques : l'exemple des Mystères de Paris d'Eugène Sue
}

\section{Translating Mixed-Race Creoleness for the Czech Lands: The Example of Eugène Sue's Les Mystères de Paris}

CÉCILE GaUthier [cecile.gauthier@univ-reims.fr]

Université de Reims Champagne-Ardenne, France

JANA KANTOŘíKová [jana.kantorik@gmail.com]

Membre associée de l'UMR Eur'Orbem (Sorbonne Université, CNRS), France

\section{RÉSUMÉ}

Le roman de Sue Les Mystères de Paris (1842-43) a constitué un événement éditorial d'une ampleur exceptionnelle et a immédiatement circulé dans toute l'Europe, Europe centrale comprise. Ce roman présente un personnage de femme fatale, Cecily, une " créole métisse » dont la supposée essence est censée être déterminée par son origine raciale, pourtant en partie invisibilisée du fait quelle peut " passer " pour blanche. On s'intéresse ici aux modalités de traduction de cette créolité métisse ambiguë, en particulier aux difficultés posées par les enjeux lexicaux de nomination et par l'écriture d'une blancheur problématique, et ce dans la traduction tchèque (anonyme) de 1862 ainsi que dans la version allemande de Moosthal (1843), conformément au contexte culturel bilingue des Pays tchèques au XIX ${ }^{e}$ siècle.

\section{MoTS CLÉS}

Métisse ; créole ; Eugène Sue ; Les Mystères de Paris ; traduction en tchèque ; imaginaire racialiste

\begin{abstract}
Sue's novel The Mysteries of Paris (1842-43) was a publishing event of exceptional magnitude that soon expanded throughout Europe, including Central Europe. This novel presents a "femme fatale" character, Cecily, a "mixed-race Creole" whose presumed essence is believed to be determined by her racial origin, which is partly invisibilised by the fact that she can "pass" for white. In this article we study the way this ambiguous mixed-race creoleness is translated, in particular the difficulties linked with the lexical issues of naming and the representations of a problematic whiteness. The texts examined include the anonymous Czech translation of 1862 as well as the German Moosthal's version of 1843, in accordance with the bilingual cultural context of the Czech lands in the 19th century.
\end{abstract}

\section{KEYWORDS}

Mixed-race; Creole; Eugène Sue; The Mysteries of Paris; translation into Czech; racial imagery

REÇU 2021-04-01 ; ACCEPTE 2021-06-15 
On connaît Les Mystères de Paris, cet extraordinaire succès éditorial des années 1840, comme roman social, dont on avance même que les thèses à résonances saint-simoniennes et fouriéristes n’auraient pas peu contribué à l’avènement de la révolution de 1848. Mais dans les premières pages c'est à une plongée exotique que le narrateur invite : les bas-fonds parisiens sont peuplés de "barbares ", de créatures aussi étrangères au lecteur bourgeois de l'époque que les "sauvages peuplades » (Sue 2009 : 35) dépeintes par Fenimore Cooper ${ }^{1}$. Parmi cette nombreuse galerie de personnages des classes populaires, considérés avec une distance quasi anthropologique, et redoublés de tout un personnel cosmopolite, il en est néanmoins deux qui viennent bel et bien de cieux exotiques, des lointaines plantations de Floride : David, le « cher docteur nègre » et Cecily, son « indigne épouse » (190), " une créole métisse » (831). Tous deux ont été arrachés à l’esclavage et amenés en Europe par le prince allemand Rodolphe, flamboyant héros vengeur du roman. Ces personnages secondaires sont l'héritage des premières amours littéraires de Sue, à savoir les romans maritimes, auxquels il s'adonna dans les années 1830, ayant lui-même, en tant que chirurgien de marine, navigué et séjourné aux Antilles². Cette migration d'un genre à un autre n'empêche nullement l'« infernale » Cecily de venir trouver sa place dans cette faune parisienne assimilable à une tératologie urbaine, fascinante et scandaleuse (Nathan 1990 : 54). A son tour ce personnage est donc appelé à circuler et se métamorphoser à travers l'Europe dans les nombreuses traductions, adaptations et réécritures qui font du livre une « matrice exceptionnelle pour la littérature mondiale ", un phénomène propre à nourrir l'écriture d'une « histoire littéraire transnationale » (Thérenty et Kalifa 2016).

Dans la perspective d'une histoire des imaginaires racialistes ${ }^{3}$ et spécialement des représentations de l'altérité noire ${ }^{4}$ dans la littérature européenne, on peut émettre l'hypothèse que ces imaginaires et représentations au XIX siècle ont plutôt circulé depuis les littératures marquées par des contextes coloniaux, comme la littérature française, vers des littératures moins exposées à cette histoire, comme celles d'Europe centrale. Les traductions, tout particulièrement, sources de transferts culturels, feraient circuler ces imaginaires, les modifiant et les ajustant, et ce de façon d'autant plus nette que l'élaboration fictionnelle de l'altérité noire éclaire avant tout sur l'imaginaire de la blancheur, une blancheur fantasmatique qui renvoie à des enjeux de construction identitaire plus ou moins conscients et formulés. Ainsi travailler sur les représentations de l'altérité noire en Europe centrale pourrait peut-être conduire à éclairer autrement des processus identitaires propres à

$1 \quad$ Les citations des Mystères de Paris sont tirées de l'édition établie par Judith Lyon-Caen (Sue 2009).

2 Il y a d'ailleurs écrit, en 1826, des Lettres sur la Guadeloupe, publiées en 1830 dans La Revue des deux mondes. Il s'y montre sensible à la souffrance des esclaves, au sujet lesquels il ne semble pas manifester de préjugé racial particulier - il les observe plutôt avec un intérêt anthropologique aigu. Pour autant il ne remet pas en cause clairement l'esclavage, s'inquiétant également du sort des colons qui ne seraient pas à l'abri de la révolte de leurs esclaves, venant s'ajouter à d'autres risques comme les ouragans ou les pénuries. Rappelons que la sensibilité « socialiste » de Sue ne s'est développée que plus tardivement. Lambiguité de ces lettres fait même écrire au directeur de la revue : « Les opinions libérales de notre jeune collaborateur sont trop connues pour qu'on puisse voir dans ces réflexions un plaidoyer en faveur de l'esclavage. » (Sue 1830:347).

3 Le racialisme désigne ici l'idéologie raciale développée au cours du $\mathrm{XIX}^{\mathrm{e}}$ siècle, théorisée dans un corpus « scientifique » mais également décelable dans les représentations collectives.

4 Peut-être conviendrait-il de recourir au terme de Blackness, utilisé notamment dans les études américaines, mais qui met surtout l'accent sur la construction identitaire, voire les revendications identitaires, des populations « noires ", ce qui n’est pas notre propos ici. Nous opterons donc pour l’expression «altérité noire ", mais en appréhendant l'altérité en tant que construction, non comme une donnée. De même, nous employons ici les termes tels que « race », «blanc» ou «noir », dans un sens critique, comme des élaborations historico-sociales. 
cette aire. Létude du personnage de Cecily s'impose à cet égard à double titre : en raison du succès inédit du roman-feuilleton, avéré aussi en Europe centrale et dans les Pays tchèques, et parce que ce personnage est un des personnages métis marquants de la littérature de langue française de l'époque (Saada 2007 : 26) - littérature populaire, en l'occurrence, qui par sa large diffusion joue un rôle significatif dans l’évolution des imaginaires collectifs, qu'elle les reflète ou les modifie. La question du métissage est cruciale dans la réflexion sur les imaginaires racialistes car il en met au jour les secrètes hantises : fantasme d'une pureté raciale nettement cloisonnée, horreur d'une " noirceur » invisible, d'un stigmate non-identifiable. Au cœur de l'élaboration textuelle du personnage se loge de fait cet enjeu de problématique blancheur, dans la mesure où Cecily " passe » pour blanche ${ }^{5}$ et que le ressort de l'action qu'elle mène est conditionné par cette aptitude, hautement romanesque mais tout aussi inquiétante, au déguisement. Les procédés d'écriture et de traduction de la «créolité métisse » du personnage sont divers : explicite de la nomination, déploiement d'un discours racialiste, recours à des images et schèmes narratifs stéréotypés, mais aussi processus peut-être moins conscients, notamment dans l'élaboration d'un imaginaire de la blancheur.

Il s'agira donc ici d'examiner les formes et modalités de traduction de cette altérité noire perçue comme trompeuse, par l'examen d'un corpus de quatre traductions tchèques des $\mathrm{XIX}^{\mathrm{e}}$ et $\mathrm{XX}^{\mathrm{e}}$ siècles ${ }^{6}$. Nous croiserons les lectures, mais accorderons une attention privilégiée au premier texte de 1862. L'histoire des traductions dans les Pays tchèques étant prise dans une configuration triangulaire, il conviendra d'y adjoindre la traduction allemande de Erwin von Moosthal, publiée dès $1843^{7}$, et qui semble avoir constitué le texte de référence pour la première traduction tchèque. Nous suggérerons ici quelques pistes et hypothèses, lesquelles demandent à être développées à une plus large échelle, en tenant compte justement de cette situation de transferts culturels triangulaire (au minimum) qui vient complexifier l'analyse de cette circulation des imaginaires racialistes.

\section{La créolité métisse de Cecily dans Les Mystères de Paris : formes et fonctions}

D’emblée, Cecily est présentée comme une "métisse » : un examen plus approfondi des usages de ce terme sera mené lors de létude comparative des traductions. Notons dès à présent que cette désignation, héritée des classifications raciales peu à peu élaborées depuis le $\mathrm{XV}^{\mathrm{e}}$ siècle, dans le contexte de la première colonisation européenne, suppose, ce qui n'est pas sans soulever les premières contradictions inhérentes à cette question, qu'elle est perçue comme un être hybride, issu de races différentes, mais que dans le même temps elle est située socialement du côté noir, non blanc, eu égard à l'appréhension strictement binaire de la question raciale largement diffusée à partir des sociétés esclavagistes et ségrégationnistes. Cette ambiguité fondatrice fait que «l'individu métis

5 Rappelons que le "passing ", en contexte nord-américain, consiste, pour un personnage très clair de peau mais assigné à une identité raciale noire, à franchir la «Color line » et se "faire passer " pour blanc. Ce procédé nourrit de nombreuses fictions, dites fictions du passing.

6 Voir la bibliographie en fin d’article. Il existe encore une traduction de S. T. Eifer, dans les années 1930, que nous n'avons pas pu consulter.

7 Voir également la bibliographie. Nous citerons aussi ponctuellement Die Geheimnisse von Paris, Deutsch von Dr August Diezmann, Leipzig, Wigand, 1844. 
[est] d’emblée désigné à la fictionnalisation » (Bourse 2017 : 39) : à la fois parce que ses origines complexes interrogent et soulèvent le soupçon du mystère et souvent de la violence, et parce que son statut « entre deux » le voue à une trajectoire non linéaire. Dans le cas d'individus si clairs de peau qu'ils peuvent passer pour blancs, la propension au romanesque est portée à son comble, puisque toutes sortes de jeux sur l'identité sont rendus possibles.

Or c'est le cas de Cecily, qui dans le roman est amenée à jouer le rôle d'une servante alsacienne, avec costume régional de circonstance. Elle apparaît même comme un pur élément du romanesque : elle n'a pas de profondeur en tant que personnage mais est un instrument au service de l'intrigue, au sens propre du terme dans la mesure où elle ne sert qu'une seule action, la vengeance que Rodolphe veut exercer à l'encontre du notaire Ferrand. Ce monstre libidineux, violeur sans scrupules, voleur et assassin insoupçonné, doit être puni par là où il pèche, à savoir la luxure. Cecily est réduite au statut d’appât, Rodolphe réussissant à la faire engager chez Ferrand comme servante, après l'avoir fait revenir d’Allemagne où cette femme sensuelle et dépravée avait été emprisonnée pour avoir trahi son époux. Tout au long de l'intrigue, de loin en loin, Cecily est attendue, son arrivée et les premiers effets qu'elle suscite sont commentés, bien avant que le lecteur ne la voie entrer vraiment en scène. Car les sulfureux « chapitres de Cecily », comme les nomment parfois les critiques, se résument finalement à deux grandes scènes, qui sont aussi deux grands tableaux : la longue scène de séduction, au cours de laquelle elle aguiche Ferrand qui la contemple à travers un " guichet », une petite fenêtre ouverte dans la porte de sa chambre; et la tout aussi longue scène d’agonie du notaire, lequel, floué par Cecily qui a fui sans se donner à lui, finit par mourir littéralement de désir. Notons que la jeune femme dans ce second tableau napparait plus que dans les visions de Ferrand. Ainsi tout au long du texte elle est moins une présence qu'un fantasme. Le mystère est en outre exacerbé par le fait que le lecteur ne comprend qu’à la fin l'" usage » que Rodolphe veut faire d'elle.

Ce qui la détermine, c'est sa beauté hors du commun, forçant l'admiration de tous ceux qui la croisent, envoûtés surtout par ses yeux magnétiques, son regard presque irréel de noirceur, et qu'il faut interpréter comme un stigmate racial : sa pupille est si large qu'elle envahit le blanc de l'œil (869). C’est une beauté sensuelle et provocante, qui n’a rien à voir avec la beauté pure (car aristocratique ?) de Fleur-de-Marie, pourtant tombée par misère dans la prostitution, et qui s'avère nêtre autre que la propre fille de Rodolphe, qu'il cherchait depuis des années. Brynja Svane remarque le traitement singulier que réserve l’auteur à Cecily, et qui la rapproche de Sarah, première épouse de Rodolphe, femme dévorée d’ambition et mère dénaturée qui a abandonné leur fille :

La solidarité que Sue montre à l'égard des femmes ne se dément donc pas. Qu'il soit question de prostitution, de viol, d'avortement, de mariage malheureux, d’ennui ou de maternité, sa sympathie va toujours à la femme. Pourvu, toutefois, que celle-ci ne soit pas un monstre de sensualité, comme Cecily, et qu'elle n'ait pas perdu, comme Sarah, le droit à la protection d'un homme par sa trop grande cruauté et son attitude calculatrice. (Svane $1988: 251$ )

Cecily et Sarah ont en commun d’être des femmes fatales, dangereusement séductrices et néfastes pour les hommes qui les désirent, davantage monstres que femmes. L’exotisme de la femme fatale est certes souvent inclus dans le cliché, néanmoins la créolité métisse de Cecily semble déterminante dans la compréhension du personnage : elle oriente à bien des égards la perception de sa beauté extraordinaire comme étant une beauté monstrueuse. 
Il s'agit en effet d'une beauté « noire », mais qui ne se révèle pas, ou ne se «trahit » pas, comme telle : donc une beauté jugée suspecte, impure, qui détonne en outre dans le système axiologique de la littérature populaire où lêtre est le plus souvent en adéquation avec le paraître, donc les méchants identifiables par leur laideur. C’est pourquoi sa beauté fait peur : " Une âme perverse sous de gracieux dehors me cause toujours une double horreur " (190), avoue l'un des proches de Rodolphe, ce que l'on peut comprendre, en le transposant sur le plan de l'imaginaire racialiste, comme un aveu apeuré de ce que les stigmates de la noirceur (morale et raciale) ne sont pas assez visibles chez elle, ce qui empêche d'être sur ses gardes.

On remarque précisément tout un jeu trouble sur la « monstration » de son corps, notamment dans la scène du guichet où elle se donne à voir à Ferrand. Quand bien même Cecily est censée être enfermée volontairement dans sa chambre, et pouvoir ouvrir et fermer le guichet à sa guise, telle une dominatrice disposant de son corps qu’elle soustrait au désir masculin, ce dispositif spatial n'est pas sans analogie avec les scènes d'exhibition, au début du XIX ${ }^{\mathrm{e}}$ siècle, des hommes et femmes " sauvages » (on pense à Sawtche, la " Vénus hottentote »), devenant à la fin du siècle des spectacles de masse, de véritables zoos humains ${ }^{8}$. Dans la scène du guichet, Cecily joue de l'exotisme de son costume, s'allonge langoureusement pour chanter une chanson d'amour de son pays (l'érotisme de la voix est très accentué), mais surtout elle se donne à admirer en exhibant son corps avec une insistance étrange : « Regardez-moi bien en face [...] Quoique vêtue en paysanne alsacienne, estce que j’ai l'air d'une servante. [...] Et ce pied, est-ce un pied de servante ?» (873)

Elle indique ouvertement que l'apparence est trompeuse et que son identité est autre : « Et qui êtes-vous donc? » balbutie Ferrand, qui fasciné, ne peut pourtant s'empêcher de regarder. Le procédé est répété dans le chapitre suivant, mais c'est cette fois la blancheur, gage de beauté, qui est objet de la provocation :

- Chaque jour je vous trouve plus belle encore.

- Et mon bras voyez donc comme il est blanc.

- Monstre... va-t’en ! va-t’en !... sécria Jacques Ferrand furieux. » (878)

La réaction violente et impulsive de Ferrand semble lourde d'un impensé : Cecily est-elle accusée de monstruosité du fait de son jeu pervers ? Ou le notaire manifeste-t-il là l'horreur suscitée par la vision de ce bras blanc et exhibé en tant que tel, comme arme de séduction, alors même que cette blancheur est jugée impure ? Il y a ici comme une provocation, un appel à voir autre chose, qui serait la noirceur sous-jacente, « essence » véritable du personnage, et source du scandale.

Il n'est pas anodin que ce personnage soit féminin. La monstruosité est en effet aussi celle de la sexualité, une sexualité perçue comme incontrôlable, "sauvage ", celle-là même que l’on croit deviner à l'origine de l'individu métis, et à laquelle il s'adonne à son tour - il, ou plus exactement elle, la femme métisse, doublement objet de méfiance, pour sa race et son sexe (dans le double sens de " genre » et de "sexualité »). Cette sauvagerie est traduite par l'omniprésence de la métaphore animale dans le texte, qui engendre la métamorphose de l'homme en bête, et du maître en esclave : Ferrand devient le «tigre » de Cecily (elle-même tigresse et serpent), acceptant de la servir et de se traîner à ses pieds. Il abdique sa volonté et sa place dominante dans la hiérarchie sociale, genrée 
et raciale. Rappelant l'ancrage littéraire et religieux de cette représentation de la femme métisse, Alexandra Bourse analyse cette "sexualité excessive » comme " le lieu de la monstruosité des personnages métis » (131), en tant qu'il est agent de destruction. Ce schème destructeur est patent dans Les Mystères de Paris, puisque Ferrand meurt de son désir pour Cecily, et il se voit en outre déployé et explicité : le texte se fait pédagogique, le narrateur déployant un discours racialiste, supposé objet de croyance partagé.

Disons-le, cette grande créole, à la fois svelte et charnue, vigoureuse et souple comme une panthère, était le type incarné de la sensualité brutale qui ne s’allume qu’aux feux des tropiques.

Tout le monde a entendu parler de ces filles de couleur pour ainsi dire mortelles aux Européens, de ces vampires enchanteurs qui, enivrant leur victime de séductions terribles, pompent jusqu’à sa dernière goutte d’or et de sang, et ne lui laissent, selon l'énergique expression du pays, que ses larmes à boire, son cœur à ronger.

Telle est Cecily. (869-870)

Cette propension à circonscrire de façon si cloisonnée la nature du personnage, qui relève de la stéréotypie propre à la littérature populaire, rappelle aussi la démarche classificatoire de l’anthropologie naissante. Elle ramène toujours davantage le personnage à sa seule fonction, mise en mouvement par un déterminisme biologique. A l'instar de David chargé par Rodolphe de crever les yeux du Maître d'école, afin qu'il découvre la faiblesse et puisse expier ses fautes en les méditant sans relâche, Cecily s'avère l'agent au féminin du châtiment infligé à Ferrand. Châtiments pour le moins cruels, même sauvages, mais dont la sauvagerie émane de Rodolphe, brouillant de façon trouble car non avouée les lignes entre barbarie et civilisation.

Mais les critiques n'ont pas manqué de s'indigner de tels excès, conférant finalement à la silhouette de Cecily dans le roman une place singulière : d'un côté elle semble invisibilisée et n'est guère pensée comme personnage (de façon significative elle napparaît quasiment jamais dans la liste des personnages incontournables du roman, bons ou mauvais) mais de l'autre elle est un rouage nécessaire de la punition et surtout elle est par excellence objet de scandale, comme le confirment les protestations suscitées par les chapitres où elle apparaît, rarement omis par les détracteurs de Sue. Le critique Nettement s’indigne en 1844 de l'immoralité de l’ouvre : «On ne persuadera jamais à un homme de bon sens qu'on ait pu, sans insulter la morale publique, peindre les provocations sensuelles jetées par la mulâtresse Cicily (sic) au notaire Ferrand ${ }^{9}$. Philarète Chasles renvoie pour sa part à la décadence latine : « Jamais la luxure latine n’enfanta de tableaux plus licencieux que celui que M. Sue a tracé de la "tentation" du notaire Ferrand par la mulâtresse Cecily... ${ }^{10}$. Peut-être ont-ils lu l'article de Sainte-Beuve "Quelques vérités sur la situation en littérature » (Sainte-Beuve, 1843), un article remarqué dans lequel l'écrivain évoque l'influence du marquis de Sade sur les romanciers contemporains, au nombre desquelles il compte, tacitement, le roman de Sue ${ }^{11}$. Il est remarquable, dans ces diverses évocations, que la créolité métisse de Cecily, tout en nétant pas explicitement désignée comme élément causal de la scène, semble néanmoins

9 Cité dans Grubitzsch (1977: 53-54).

10 Cité dans Bory $(2000: 361)$.

11 Si Sainte-Beuve ne nomme pas Sue, il reconnaît dans une lettre adressée à Juste Olivier, chroniqueur suisse, qu'il avait principalement en tête Les Mystères de Paris et les scènes finales où intervient Cecily. Voir Duchet (1968: 225). 
comprise comme tel : le simple rappel de ce qu'elle est "mulâtresse » oriente l'interprétation en ce sens, de même que le fait, sans doute involontaire, mais significatif, que son nom soit systématiquement écorché par Nettement, ce qui exhibe létrangeté de cette femme littéralement innommable. En somme c'est une sensualité sadique qu'incarne cette femme, c'est-à-dire une sensualité perverse, venue d'ailleurs (un ailleurs moral et géographique), « non pas naïve comme chez nos vieux Gaulois » ${ }^{12}$ : on ne saurait mieux dire, en ces décennies marquées par la quête passionnée des supposées origines gauloises de la France, le caractère radicalement étranger à la nation française de Cecily, qui est censée déterminer sa nature et ses actions, et représenter un danger pour l'intégrité de celui qui la désire. Mais le danger, comme l'a rappelé le narrateur, est supposé menacer plus largement les Européens, et de fait le personnage fascinant qu'est Cecily trouve bien sa place dans les diverses traductions, même un peu modifiées. Il importe de rappeler le contexte de la première traduction tchèque avant de se livrer à un examen plus précis des textes et des différents choix de traduction.

\section{Le contexte historico-culturel de la première traduction tchèque}

Le roman Les Mystères de Paris fut traduit en tchèque cinq fois et connut neuf éditions, pour la plupart dans des collections accessibles et au tirage élevé. La première traduction en 1862 fut relativement tardive par rapport à d'autres traductions très rapides, notamment en anglais ou en allemand. Cependant, Sue nétait pas inconnu de la critique et du lectorat tchèques, car Les Mystères de Paris nétait pas son premier roman traduit ${ }^{13}$. De plus, les lecteurs tchèques cultivés, souvent polyglottes, notamment germanophones, avaient à leur disposition soit la version originale, soit les traductions allemandes. Tel est le cas de Jan Neruda qui dans son article "Škodlivé směry » ("Courants nocifs ») de 1859 remarque : « Mystères de Paris dokázaly mnoho dobrého a předce byly co nemravního směru kaceřovány. » (95; «Les Mystères de Paris ont fait grand bien, pourtant ils ont été pris pour une hérésie immorale. ${ }^{14}$ ). L'exemple romanesque de Sue sert Neruda dans sa défense de la littérature réaliste et son opposition à tout idéalisme dans l'art, qui, comme il constate, associe la moralité à l'aisance et l'immoralité à la pauvreté. Karel Sabina lui aussi a lu Sue dans l’original ou en allemand. Dans « Slovo o románu vůbec a o českém zvláště » (« Sur le roman en général et sur le roman tchèque en particulier »), son étude de 1858 consacrée à l'évolution du genre romanesque, notamment du roman moderne, Sabina salue le talent de l'auteur français et sa franchise dans la description de la réalité sociale ${ }^{15}$. L’édition de la première version tchèque des Mystères de Paris répond ainsi à l'horizon d'attente évoqué, celui de la littérature

12 Duchet explique que Juste Olivier, dans un article de La Revue suisse paru en juillet, reprend la définition du sadisme que lui a donnée Sainte-Beuve : le " de Sade " c’est " de la méchanceté, de la cruauté, de la perversité dans la volupté... la volupté non pas naïve comme chez nos vieux Gaulois, mais devenue méchante, nerveuse et cruelle. » (Duchet $1968: 225)$.

13 Il s'agit des romans suivants : Le Juiferrant/Věčnýžid (1850) traduit par Václav Bambas, Les enfants de l'amour/Děti lásky (1850) traduit par Karel Hradecký (pseudonyme de Ladislav Pospíšil), La bonne aventure/Prorokování osudu (1852) par Václav Filípek, $c f$. Fryčer (2008: 84-85).

14 Les traductions des citations en tchèque et en allemand sont de notre responsabilité.

15 Voir Sabina $(1858: 231)$. 
sociale. La traduction anonyme, accompagnée du sous-titre « román sociální » (roman social), est publiée chez Hugo Silber dans une collection ${ }^{16}$ dont le titre renvoie à la fonction primordiale de la littérature et de la traduction pour la vie culturelle et nationale tchèque : " Tajnosti, čili sbírka tajností hlavních měst celé země, kterou byl, hledě vůbec k tomu, co národu českoslovanskému jest užitečného a co sloužiti může k ušlechtění mravů sestavil Hugo Silber. » (1862 ; «Les Mystères ou Recueil de mystères des capitales du monde entier, établi par Hugo Silber en tenant compte de ce qui est utile à la nation tchéco-slave et peut servir à améliorer les mœurs publiques. »)

Néanmoins, malgré cette intention louable d'une utilité collective, le roman de Sue polarisait. Pour mieux comprendre son accueil mitigé, notamment la gêne ressentie par la critique patriotique protectionniste, il faut prendre en considération le rôle de la traduction dans le contexte des Pays tchèques au milieu du XIX ${ }^{\mathrm{e}}$ siècle. Une perspective assez parlante nous est offerte par létude «O překladech v novočeské literatuře » («Sur la traduction dans la nouvelle littérature tchèque ») parue en 1877 dans la revue conservatrice Osvěta. Son auteur, l'ethnographe et traducteur de l'anglais Primus Sobotka, constate que le but de la traduction est d'enrichir la nation, langue comprise : « Jimi [př̌klady] nové idey vnášejí a vštěpují se v národ, jimi udržuje se duševní spojení s ostatním vzdělaným světem a klestí se dráha pokroku i všeobecné osvětě, jimi obohacuje a vzdělává se i jazyk domácí, jelikož se do něho uvádějí nové pojmy, nové obraty i obrazy. » (Sobotka 1877 : 304 ; " [les traductions] apportent et inculquent de nouvelles idées à la nation, elles maintiennent un lien spirituel avec le reste du monde éduqué et elles ouvrent la voie au progrès et à l'éducation générale, elles enrichissent et cultivent la langue nationale en introduisant de nouveaux concepts, de nouvelles expressions et images. »). Mais pour autant tout n'est pas bon à traduire. Ainsi, Sobotka met en garde notamment contre les " écrits de divertissement » (307 ; " spisy zábavné ») de provenance allemande ${ }^{17}$ qui submergent le marché tchèque après 1850 et qu’il qualifie de toxiques : « jedovaté plody německé spekulace působí právě tak zhoubně na ducha, jako kořalka na tělo : kdo jich jedenkráte okusil, jest nenapravitelný. » (307 ; « les fruits empoisonnés de la spéculation allemande produisent un effet nocif sur l'esprit comme l'eau-de-vie sur le corps : qui les a goûtés une seule fois est irrécupérable. »). Même si Sobotka met en avant « la réciprocité slave » (308; "vzájemnost slovanská ») et appelle à privilégier les traductions des autres littératures slaves, sa position n'est globalement pas anti-germanique. Il constate en fait que le problème principal réside en ce que le lectorat cultivé lit la littérature allemande de qualité dans l’original (par exemple l’œuvre de Friedrich Spielhagen) mais que le lectorat moins éduqué est victime de mauvaises traductions de mauvais originaux. La traduction, rappelle-t-il, s'est intensifiée dans les années 1860 , et la littérature française est devenue la plus traduite ${ }^{18}$. Néanmoins, ce n’est pas toute la production française qui est appréciée de Sobotka ; l'œuvre d'Alexandre Dumas (père) est qualifiée de « napínavé sic, ale jalové plody» (309; « fruits palpitants mais vides »). Sobotka

16 Précisons que cette collection fut inaugurée par le roman Tajnosti pražské (Les Mystères de Prague), une œuvre anonyme, parue en 1861-62, et clôturée en 1868 par le roman de Josef Svátek portant le titre Tajnosti pražské (Les Mystères de Prague) également. Lexikon české literatury émet l'hypothèse que l'auteur du premier roman Tajnosti pražské était Jan Erazim Sojka (2008 : 243).

17 Sobotka se réfère aux éditions Steinhauser et Bensinger à Prague, Buschak et Irgang à Brno (Sobotka 1877 : 307 ).

18 Lalliance culturelle tchéco-française représentait traditionnellement un contrepoids à l'influence allemande. Cf. " Avant-Propos. La langue française en Bohême » dans Catalogue des ouvrages français traduits en tchèque/Přehled překladů z jazyka francouzského do češtiny (Klouček, Žižka 1889 : XVIII) et l’article (Poučová 2020). 
remarque avec ironie à propos de Sue que la valeur de ses œuvres est confirmée par le fait que les traducteurs ne voulaient même pas y mettre leur signature (309). Ceci est évidemment une exagération, car la seule traduction anonyme de Sue est celle des Mystères de Paris.

Ainsi se dessine le lectorat cible de la première traduction de ce roman en tchèque. Certes, elle répondait à une demande de littérature de divertissement pour le public moins éduqué et moins exigeant. Toutefois, et malgré la position de la critique patriotique conservatrice, les avis décrivains comme Jan Neruda et Karel Sabina ainsi que le cadre éditorial de la traduction des Mystères de Paris témoignent de l'inscription de cette traduction dans un mouvement patriotique également : d'une part, elle permet de développer le goût de la lecture en tchèque et d'autre part, elle favorise lémergence du roman social qui a eu sa dimension nationale. Car la littérature sociale refusant d'idéaliser la réalité moderne avait pour but non seulement de montrer la vie des classes sociales défavorisées mais aussi d’améliorer la société nationale ${ }^{19}$. Dépréciation et appréciation de Sue semblent alors l'une comme l'autre être nourries de l'idée patriotique et s'inscrivent, chacune à sa façon, dans la continuité du renouveau national tchèque.

\section{Nommer la créolité métisse : les enjeux lexicaux}

En ce qui concerne les traductions des Mystères de Paris en tchèque, certaines versions s'affichent ouvertement comme des adaptations, ce qui est le cas des éditions de 1929 et 1970. Mais déjà dans la version de 1862 le traducteur prend ponctuellement quelques libertés, notamment avec lordre et le titre de certains épisodes. D’une façon générale, la trame narrative relative à Cecily est maintenue dans toutes les traductions, avec certes parfois des raccourcis, et cela même dans les adaptations plus courtes - ce qui est la preuve du caractère difficilement dispensable du personnage dans la diégèse. Avant tout, soulignons que Cecily est partout bien identifiée comme « kreolka » (créole). Sa première évocation dans le roman de Sue intervient dans une conversation entre deux des hommes de confiance de Rodolphe ${ }^{20}$. L'origine de Cecily est donnée comme déterminante, puisquelle est qualifiée quasiment dès le début d' " audacieuse métisse» (Sue 2009 : 190). Mais le terme nétait visiblement pas familier aux lecteurs français de l'époque, puisqu'il appelle une note de bas de page : "Créole issue d'un Blanc et d'une quarteronne esclave. Les métisses ne diffèrent des Blanches que par quelques signes imperceptibles. » (190). Cette invisibilité raciale, qui serait donc la caractéristique principale de Cecily, est redoublée d'un commentaire explicatif dans la conversation des personnages : «Charmante... trop charmante... il faudrait l’oil impitoyable d’un créole pour découvrir le sang mêlé dans l'imperceptible nuance bistrée qui colore légèrement la couronne des ongles roses de cette métisse » (191). Seuls alors certains stigmates trahissent son " essence », mais ils ne sont pas décelables par un œil non averti. Cecily est donc d'autant plus dangereuse qu'elle avance masquée - mais pas du point de vue du lecteur. La nature diabolique et monstrueuse de cette femme est en effet mentionnée partout, cependant la fluidité de la nomination raciale, et de sa glose, est très frappante, aussi bien d'une langue à l'autre que d'une époque à l'autre.

19 Il convient de mentionner par exemple l’ouvre de Karel Sabina, Gustav Pfleger Moravský, Antal Stašek ou Jakub Arbes. 
Arrêtons-nous tout d'abord sur la question de l'acception du mot " métis » à lépoque de Sue. Les dictionnaires de la langue française entre 1820 et 1850 semblent répéter la même définition : homme né de l'union d'un Européen et d'une Indienne ou l'inverse et, au sens plus large, mélange de deux espèces. Les références explicites au contexte espagnol ne font pas défaut ${ }^{21}$. En parallèle, le mot " métis » se retrouve également dans le discours littéraire. Avant Les Mystères de Paris, le lecteur français a pu le rencontrer par exemple dans le roman de Victor Hugo Bug-Jargal de 1826. Dans une note de bas de page (Hugo 1888 : 11), l'auteur indique le mot " métis » parmi d’autres termes spécialisés en se référant à Moreau de Saint-Méry. Cet avocat originaire de Martinique nomme et délimite dans sa classification pseudo anthropologique les neuf catégories du métissage entre Noir et Blanc (Moreau de Saint-Méry 1875 : 83-86, 90-98).

Tout comme dans le cas de Hugo, la note explicative dans le roman de Sue incarne le regard scientifique de l'Europe porté sur une réalité lointaine, à découvrir. La glose apporte ainsi un savoir spécialisé - résultat de cette découverte - et éclaircit non seulement le terme obscur " métisse » mais surtout la réalité obscure qu'il désigne : l'être de Cecily. Il est possible que la démarche de nomination de ce personnage puise dans l'expérience antillaise de Sue, dans les années $1820^{22}$. Dans cette zone géographique, le mot " métis » désignait non seulement les descendants des Blancs et des Amérindiennes des Caraïbes, mais il a été transféré également à l’axe Blanc - Noir. Progressivement, les nominations relatives au métissage se sont multipliées et ont souvent changé de signification d'une zone à l'autre, ainsi que d'une puissance coloniale à l'autre. Malgré la polysémie gagnée par le terme "métis », on constate que la définition apportée par Sue correspond au classement typique de Saint-Domingue qu’on trouve chez Moreau de Saint-Méry. Il est ainsi possible que Sue et Hugo aient partagé la même référence, à savoir son traité Description topographique, physique, civile, politique et historique de la partie française de l'Île Saint-Domingue de 1797.

La précision «scientifique » de Sue catégorisant Cecily comme «métisse » la distingue en outre de la « mulâtresse », qui parait être le terme prédominant à lépoque en métropole pour désigner l'enfant issu de la rencontre entre individus blancs et noirs. Toutefois, ce terme semble être inexact pour saisir la particularité physique de Cecily, c'est-à-dire sa blancheur suspecte. Le mot le plus fréquent dans Les Mystères de Paris pour la nommer est néanmoins "créole », employé dans des sens fluctuants. D’abord, il désigne l'habitant des « colonies », quelle que soit sa couleur de peau. Quant à Cecily, elle est nommée « créole métisse » pour bien faire comprendre au lecteur que sa blancheur n'est pas celle des Blancs. Finalement le terme "métisse » disparaît dans la suite du texte, et le mot « créole ", employé avec une grande fréquence, semble être suffisant pour rappeler la cause de ses comportements.

Avant de nous pencher sur la première traduction tchèque des Mystères de Paris, regardons les possibilités de la langue tchèque du XIX ${ }^{\mathrm{e}}$ siècle pour nommer un être « hybride ${ }^{23}$. Outre « kreol/

21 Ainsi, Le Nouveau dictionnaire de la langue française de 1820 (Chez Deterville) indique sous l'entrée métis.isse (adj) : «De l'espagnol mestizo, dérivé du latin mixtus mélangé. Nom que les Espagnols donnent aux enfans nés d'un Espagnol et d'une Indienne, ou d'un Indien et d'une Espagnole. - Il se dit aussi des chiens qui sont engendrés de deux espèces, comme d'une épagneule et d'un barbet, un chien métis; et en botanique, des fleurs et des fruits nés du mélange de deux espèces. - Il s’emploie aussi substantivement. Un métis. » (1820 : 148).

22 Ce qui suggère également l'occurrence du mot " câpresse " dans Les Mystères de Paris, typique notamment de la Guadeloupe. Cf. (Régent : 2011).

23 Notre recherche se base sur les occurrences enregistrées dans les archives de Příruční slovník jazyka českého (PSJČ) https://psjc.ujc.cas.cz/. La limite inférieure des données collectées est 1770. 
$\mathrm{ka}$ » pour « créole», « mulat/ka » pour « mulâtre »/ mulâtresse » ou « s/míšenec »/ s/míšenka » pour désigner des «croisements» de toute sorte, on peut rencontrer également les termes pseudo anthropologiques précisant le degré du métissage comme "terceron/ka », " quarteron » ou " quinteron ». De surcroît, ce vocabulaire issu des puissances coloniales avait même ses équivalents tchèques comme " třetík » / " třetice ", "čtvrtík » et " pětík » ${ }^{24}$ répondant ainsi aux désirs linguistiques patriotiques de créer sa propre terminologie en langue nationale. Le tchèque du XIX siècle connaissait même le mot «mestic/ka » en tant quéquivalent de " métis »/ "métisse ». On peut alors s'interroger sur la raison pour laquelle le traducteur en 1862 n'a pas traduit " métisse » par « mesticka " mais par « kreolka ». Une des raisons probables est le fait que le mot " mestic ", d'ailleurs assez rare, était associé uniquement à l'Amérique latine, notamment au Mexique. Ainsi, Riegriov slovník naučný, la première encyclopédie tchèque (1860-1874, officiellement sous-titrée Slovník naučný) propose la définition suivante, qui semble en définitive assez éloignée de l'image de Cecily chez Sue : "V Mexiku jest mnoho m-ců, jichž plet’ je téměř čistě bílá, prohledná, brada řídká, ruce a nohy malé, oči šikmé. Ženy slovou mestizas, a jich děti s bělochy liší se nepřiliš od Evropanův. » (1866 : 264 ; «Il existe de nombreux métis au Mexique, dont la peau est presque d'un blanc pur, est claire, le menton fin, les bras et les jambes petits, les yeux bridés. Les femmes sont nommées mestizas, leurs enfants avec des Blancs ne différent pas trop des Européens. ») ${ }^{25}$.

Comment alors traduire en tchèque la créolité métisse noire qui est invisible ? Le mot " mestic» est intrinsèquement lié au mot « kreol », car tous les métis étaient - en principe - créoles. En revanche, tous les créoles nétaient pas métis. Slovník naučný ${ }^{26}$ indique que les « kreolové » sont des descendants européens blancs nés aux États-Unis, mais, au sens plus large, il s'agit aussi de « lidi všech barev» (1865: 987 ; " personnes de toutes les couleurs »). L'ambiguïté du mot invite nécessairement aux fantasmes. De plus, comme l’encyclopédie nous en informe, «ženštiny [kreolské mají] nejkrásnější oči ze všech na světě, veliké, nyvé, výrazu plné, i zuby mají pěkné » ( 987 ; « les femmes [créoles ont] les plus beaux yeux du monde, grands, attendrissants, très expressifs, et même leurs dents sont belles »). Il semble que les possibilités pour nommer la créolité métisse noire invisible aient été restreintes : le mot "mulatka » était hors de question pour la visibilité de la "noirceur", les termes comme " quinteron " ou plutôt " quinteronka " (catégorie correspondant au cas de Cecily) probablement inadaptés car considérés trop « scientifiques » pour un roman populaire. Le sens du mot «s/míšenka » semble être au XIX ${ }^{\mathrm{e}}$ siècle assez large et flou ${ }^{27}$. Dans ce sens, on peut se demander pourquoi le traducteur tchèque n’a pas suivi la démarche de Sue et n’a pas traduit le mot

24 Les archives de PSJČ indiquent comme source la revue Krok. Veřejný spis všenaučný pro vzdělance národu českoslovanského de 1831 avec l'indication «(A. Jungm.) ». Il est possible que l'auteur de cette terminologie soit le médecin Antonín Jan Jungmann, frère du philologue et lexicographe Josef Jungmann.

25 Le tome de Slovník naučný consacré à la lettre M fut publié en 1866. Les occurrences enregistrées dans les archives de PSJČ qui précédent cette date se réfèrent également plutôt à l'Amérique latine. En revanche, Pavel Josef Šafařík dans Slovanské starožitnosti (1837) emploie le mot " mestic " pour évoquer le contexte de la Grèce et la population mélangée autour des villes grecques.

26 Cf. l’entrée "Kreolové » dans Slovník naučný (1865). Les occurrences enregistrées dans les archives de PSJČ témoignent de cette ambiguïté du mot : par exemple « vedle bílé Kreolky, dcery evropských rodičů, vidíme mouřenína »// "à côté d'une créole blanche, fille de parents européens, on voit un Noir » (Obzor, 1855).

27 Slovník naučný ne l'indique pas du tout, quant aux occurrences dans les archives de PSJČ, on trouve le sens de "bâtard ", hybride botanique et zoologique, rarement " métis " au sens large ou au sens de " mulâtre ». Le mot « pomíšenec » signifie plutôt « renégat ». Quant à Ottův slovník naučný (1901), il applique le mot « míšenec » uniquement au croisement des animaux. 
avec une glose comme l'a fait le premier traducteur de l'allemand Erwin von Moosthal. Il semble que pour le traducteur tchèque, le souci informatif dans ce domaine nétait pas au premier plan, voire était jugé inutile pour le lecteur tchèque, et que le mot «kreolka » paraissait vraisemblablement assez exotique. En revanche, la première traduction allemande témoigne d'un intérêt pour le classement et l'enrichissement du savoir, et la note qui accompagne le mot " Mestize " amplifie ainsi la dimension encyclopédique : elle propose une explication beaucoup plus poussée que l'original, en apportant des détails sur l'apparence physique d'un groupe anthropologique ${ }^{28}$.

\begin{tabular}{|c|c|}
\hline Texte de Sue & $\begin{array}{l}\text { «cette audacieuse métisse » (190) } \\
\text { Note: «Créole issue d'un Blanc et d'une quarteronne esclave. Les métisses ne } \\
\text { diffèrent des Blanches que par quelques signes imperceptibles. }\end{array}$ \\
\hline $\begin{array}{l}\text { Traduction } \\
\text { allemande } \\
\text { de } 1843\end{array}$ & $\begin{array}{l}\text { «diese kühne Mestize» (35) } \\
\text { Note : « Mestizen, im Spanischen mestizos, nennt man in Amerika diejenigen } \\
\text { Creolen, welche von einem Europäischen Vater und einer Quarteronne, dem } \\
\text { farbigen Abkömmling von Europäern und Weißen im fünften Glied oder } \\
\text { umgekehrt, abstammen. Sie haben gewöhnlich eine röthere Gesichtsfarbe als die } \\
\text { Europäer, und wenig Barthaare, dunkle Nägel und noch etwas vom Negerauge, } \\
\text { sonst unterscheiden sie sich in Nichts von den Europäern*». }\end{array}$ \\
\hline $\begin{array}{l}\text { Traduction tchèque } \\
\text { de } 1862\end{array}$ & $\begin{array}{l}\text { " tato smělá kreolka » (244) } \\
\text { Absence de note }\end{array}$ \\
\hline $\begin{array}{l}\text { Traduction tchèque } \\
\text { de } 1924\end{array}$ & $\begin{array}{l}\text { "tato smělá míšenka » (54) } \\
\text { Absence de note }\end{array}$ \\
\hline $\begin{array}{l}\text { Traduction tchèque } \\
\text { de } 1929\end{array}$ & Omission de ce passage \\
\hline
\end{tabular}

* «En Amérique on appelle métis, en espagnol mestizos, les créoles qui descendent d'un père européen et d'une quarteronne, la descendante de couleur des Européens et des Blancs à la cinquième génération, ou inversement. Ils ont généralement un teint plus cuivré que les Européens, sont imberbes, ont des ongles foncés, et encore dans l'œil quelque chose du nègre, sinon ils ne diffèrent en rien des Européens. »

Ce que l'on peut constater, c'est que le mot " mesticka " n'apparaît dans aucune traduction des Mystères de Paris en tchèque. Il est resté spécialisé, appartenant à un contexte historique précis, toujours lié plutôt aux Amériques. Quant au mot « kreolka » choisi par le premier traducteur tchèque, il contribue directement à la bizarrerie indéfinissable de Cecily. En effet, cette nomination, qui est vague en termes de couleur de peau mais identifie le personnage comme exotique, appartenant à l'univers colonial, est favorable à la con-fusion présente dans l'aspect physique de cette ancienne esclave de Floride, aux bras blancs, qui passe sans problème pour une Alsacienne. Enfin, on peut imaginer que le mot « kreolka » a pu créer un effet réussi d'immersion involontaire, car le manque d'intérêt informatif et de "rigueur " pseudo anthropologique que son choix représente met finalement les lecteurs tchèques, non instruits, dans la position des personnages des Mystères de Paris qui rencontrent pour la première fois Cecily, cette femme paradoxale, masquée, blanche et noire.

28 Cf. des éditions de Brockhaus des années 1830 et 1840 (1832 et 1843), où le mot « Mestiz » figure uniquement dans l'entrée «Amerika » et désigne l'enfant né de l’union Européen - Amérindien (1832: $71 ; 1843$ : 290). 


\section{La noirceur « invisibilisée » dans les traductions tchèques?}

L'impression qui ressort de l'examen des traductions tchèques, quant à l'écriture de la créolité métisse, est d'une forme d'atténuation, peut-être d'invisibilisation redoublée, quoique sans déni ou censure, de cette noirceur cachée. Dans le texte de Sue Cecily passe pour blanche, aux yeux d'un observateur non averti, mais le narrateur et les personnages informés ne cessent de rappeler au lecteur qu'elle est "créole ». Si Cecily est bien aussi qualifiée de « kreolka » dans les traductions tchèques, incitant le lecteur à interpréter son comportement à l'aune de ses origines raciales, le retour obsessionnel de cette nomination semble néanmoins un peu moins marqué. On remarque surtout, ce qui relève pour le coup bel et bien de l'effacement, la disparition de bon nombre dénoncés explicitement racialistes, telle la longue définition de Cecily comme incarnation de « la sensualité brutale qui ne s'allume qu'aux feux des tropiques », que le traducteur de 1862 omet. Cela vaut aussi pour la traque des stigmates raciaux, comme la couronne des ongles censée trahir l'essence noire. Cet élément n'est pas plus traduit lors de la première évocation du personnage que dans la citation suivante, tirée de la scène du guichet : "Et elle montra une main blanche, charmante, aux doigts fins et déliés, aux ongles roses et polis comme de lagate, mais dont la couronne légèrement bistrée trahissait le sang mêlé » (873). Beauté blanche et stigmate racial coexistent de façon suspecte : c'est précisément cette mention de la couronne bistrée qui rend à rebours la blancheur suspecte. Mais cet indice racial, pourtant bien traduit en allemand, disparait de toutes les traductions tchèques, ainsi en 1862 : «Podívejte se na mou ruku, dí dále, a věru, ruka její byla jako alabastrová...» (217; «Regardez ma main, ajouta-t-elle, et, en effet, sa main était blanche comme l'albâtre »). Non seulement le traducteur supprime la mention des ongles, mais il prête au narrateur (par l'adverbe « věru ») la volonté de souligner la blancheur des mains en ajoutant une comparaison convenue avec l'albâtre. Dans les traductions des années 1920, les ongles sont certes décrits, mais sans particularité autre que leur couleur rose. En revanche la main donne de nouveau lieu à des qualifications fluctuantes, et si sa blancheur est mentionnée dans le texte de 1924 ("A ukázala mu bílou půvabnou ruku s něžnými prsty a růžově zbarvenými nehty » (167), « Et elle lui montra une charmante main blanche, aux doigts fins et aux ongles de couleur rose »), elle disparaît de la traduction suivante, pourtant très proche : «A ukázala mu malou, půvabnou ruku s něznými prsty a růžově zbarvenými nehty» (1929:66; «Et elle lui montra une charmante petite main, aux doigts fins et aux ongles de couleur rose. »).

Ces exemples, dont la lecture invite à la prudence interprétative, n’en illustrent pas moins une récurrence du texte qui est la singularité de l'imaginaire de la blancheur dans le texte de Sue, eu égard aux descriptions du corps de Cecily : celles-ci, dans leur ambiguïté, viennent déstabiliser l'explicite de la nomination - à moins qu'elles ne déploient justement toute l'ambiguïté de cette nomination, s'appliquant à des personnages hybrides. Il faut préciser que la blancheur du personnage est indéniable : son visage est "pâle» $(855,869)$, même « incolore » $(869)$, ce qui contraste en outre de façon frappante avec ses lèvres rouges, ses yeux et cheveux noirs (869) - autant d'éléments généralement bien présents dans les traductions. Mais c'est une blancheur étrange, "mate » (869), marquée par certaines particularités que l’on ne retrouve pas toujours dans les textes tchèques, ainsi de la "blancheur éblouissante, mais sans transparence » de son " cou élégant et potelé » (869) que le traducteur de 1862 n’a pas traduit, contrairement à Moosthal. Comme il a été précisé, elle est aussi exhibée à la vue du personnage, et du lecteur. Ainsi l'épisode de « monstration » du 
bras blanc est bien perçu comme un moment significatif de la relation entre les deux personnages puisqu'il a été conservé dans toutes les traductions, et ce alors même que la scène du guichet est longue et a donné lieu à des coupes dans les versions adaptées. On remarque cependant que le sous-texte inconscient semble bien souvent modifié ${ }^{29}$.

\begin{tabular}{|l|l|}
\hline Texte de Sue & $\begin{array}{l}\text { - Chaque jour je vous trouve plus belle encore. } \\
\text { - Et mon bras voyez donc comme il est blanc. } \\
\text { - Monstre... va-t'en ! va-t’en !... sécria Jacques Ferrand furieux. }\end{array}$ \\
\hline $\begin{array}{l}\text { Traduction } \\
\text { allemande de 1843 }\end{array}$ & $\begin{array}{l}\text { « Wie gefällt Ihnen mein Arm ? Es ist rund und weiß (rond et blanc), nicht wahr?” } \\
\text { fragte Cäcilie neckend. } \\
\text { - Ungeheuer (monstre) ! laß mich! Geh! » rief Jakob Ferrand wie wüthend. » (152) }\end{array}$ \\
\hline $\begin{array}{l}\text { Traduction tchèque } \\
\text { de } 1862\end{array}$ & $\begin{array}{l}\text { « Opravdu? A jak se vám líbí mé rámé? Okrouhlé (rond), není-li pravda? » } \\
\text { « Obludo (monstre) ! nech mne...tíhni! zvola Jakub Ferrand celý zběsilý. » (222) }\end{array}$ \\
\hline $\begin{array}{l}\text { Traduction tchèque } \\
\text { de } 1924\end{array}$ & $\begin{array}{l}\text { "A pohled’te, jak bílé (blanc) jsou mé paže! » } \\
\text { « Netvore (monstre), nepokoušej mne (ne me tente pas)! » zvolal pološíleně } \\
\text { Ferrand. (176) }\end{array}$ \\
\hline $\begin{array}{l}\text { Traduction tchèque } \\
\text { de } 1929\end{array}$ & $\begin{array}{l}\text { A pohled’te, jak oblé (rond) jsou mé paže ! » } \\
\text { «Pust'te mě dovnitř. Neublížím vám. Vždyt jsem poslušný jako jehňátko. » (70) }\end{array}$ \\
\hline
\end{tabular}

En effet, dès la traduction de Moosthal, et conformément à sa tendance au redoublement des termes, le bras de Cecily est qualifié de " rund und weiß", " rond et blanc ", suscitant la fureur de Ferrand qui traite la jeune femme de " ungeheuer ", " monstre ». La traduction de 1862 laisse bien apparaître la proximité avec le texte allemand plutôt qu'avec le texte original ( "Wie gefällt Ihnen »// " jak se vám líbí », c’est-à-dire " vous plaît-il ?/que pensez-vous de ? ; ou encore « nicht wahr »// " není-li pravda », c'est-à-dire "n’est-ce pas »). Cecily est également qualifiée de « obluda", "monstre ». On est cependant étonné de voir s’effacer, par le truchement de la traduction allemande, la qualification de blancheur, au profit de la seule rondeur du bras. Si la mention de la couleur revient, et de façon exclusive, dans le texte de 1924, on remarque que l'exclamation de Ferrand perd en revanche de son ambiguité interprétative par l'ajout d'une glose (« ne me tente pas!»), qui fait porter l'accusation de monstruosité plus nettement sur la provocation perverse de Cecily que sur la possible horreur suscitée par l'exhibition de cette blancheur suspecte mais fascinante. La scène, raccourcie dans l'adaptation de 1929, perd de sa violence, et l'accusation de monstruosité disparaît, de même que le motif du bras blanc. L'interprétation de ces divers exemples reste certes malaisée, mais on peut émettre l'hypothèse que les traducteurs ne sont pas insensibles à la bizarrerie de cette blancheur. Les choix de traduction, même opposés (tantôt un renforcement du motif, tantôt une modification ou un effacement) pourraient être compris comme le signe d'une perception plus ou moins consciente de ce que se nouent en ces endroits précis du texte des enjeux importants pour la compréhension du personnage problématique qu’est Cecily.

29 A l'exception de la traduction de Diezmann, qui reste là encore très littérale : « - Und, sehen Sie nur, wie weiß mein Arm ist. - Ungeheuer - hebe Dich weg von mir! » (27). 
Un dernier point à observer concerne la traduction de l' « étrange et effroyable maladie » (1042) entraînant la mort du notaire, maladie de nature scandaleuse, car explicitement sexuelle. Elle est causée par " une frénésie érotique » (1042) qui provoque une exacerbation de chaque sens, dès lors soumis à des tortures inhumaines. Une note en précise le nom savant, faisant appel à diverses autorités médicales :

[...] Voir aussi les admirables pages d'Ambroise Paré sur le satyriasis - exacerbation maladive (morbide, pathologique) du désir sexuel chez l'homme -, cette étrange et effrayante maladie qui ressemble tant, dit-il, à un châtiment de Dieu... (1042)

Il y a là un lien plus étroit qu’il n'y paraît avec Cecily, qui est clairement identifiée - elle, en tant qu' " infernale créole $»^{30}$ - comme l'agent de la maladie, dévoilant un imaginaire de la contamination raciale qui se propagerait par la sexualité, rendant possible le mélange des races si redouté. Le regard porté sur le personnage s'avère tout du long en grande partie scientifique, spécialisé - que ce soit anthropologique et/ou médical.

La maladie de Ferrand est bien présentée dans la traduction tchèque de 1862 comme "šílená smyslná žádost » (113; " un désir sensuel dément »), et le schème narratif de dégradation physique et psychique est bien décliné jusqu'à la spectaculaire scène finale d'hallucination, au cours de laquelle le notaire se traîne par terre en se prenant pour un tigre. Néanmoins le tableau d’ensemble est atténué. On ne trouve pas de note de bas de page précisant l'étrange nom médical de " satyriasis» (alors que la maladie est bien ainsi nommée dans les traductions des années 1920), et si le lien causal avec Cecily est avéré, elle ne se voit pas qualifiée, au début du chapitre «Furens amoris ", d' " infernale créole » mais de "d'ábelská žena " (112; "femme diabolique »). Surtout l'évocation joue beaucoup moins sur l'effroi, là où Sue (dont le goût pour les excès et monstruosités, dans Atar-Gull, avait déjà été dénoncé par les critiques) décrit non sans complaisance les longues et atroces souffrances du notaire. On ne retrouve pas les « rugissements sourds et inarticulés » (1024), le cri « qui n’avait rien d'humain » (1049), les " atroces convulsions » (1051) et autres « contorsions surnaturelles» (1052) qui conduisent à la mort du notaire. Moosthal en revanche ne craint pas dêtre fidèle à ce tableau glaçant.

Cette atténuation dans le texte tchèque - qui ne signifie pas pour autant une disparition du motif - pourrait être le pendant final de l'atténuation, au début du roman, de la dépravation morale de Cecily, comme s'il s'agissait d'amoindrir un double scandale moral et esthétique. Le texte de Sue apparaît en effet, aux yeux également de nombre de lecteurs français, comme monstrueux par ses excès, excès dont Cecily constituerait l'une des formes, certes pas la seule mais l'une des plus incompréhensibles ou équivoques, dans la mesure où sa monstruosité invisible ne se prête pas à une herméneutique rassurante. Cette atténuation, dans et par la traduction, pourrait être une façon, tout en conservant la trame narrative essentielle, de dompter cette sauvagerie.

Traduire en tchèque l'invisibilité de la créolité métisse noire dans Les Mystères de Paris représentait évidemment un défi, mais la force de cette «matrice littéraire »l'a imposée comme traduisible, tout en obligeant les traducteurs à se confronter à l'étrangeté des termes raciaux toujours plus

30 " il y a aujourd'hui six jours... que l'infernale créole a allumé le feu inextinguible qui dévore cet homme... ", remarque le médecin complice de Ferrand (1042). 
présents dans la littérature des pays coloniaux. Dans la traduction de 1862 privilégiée dans cette étude, on a pu constater que, si ce motif était bel et bien inscrit dans la traduction, il apparaissait à la fois présent et absent, mentionné mais peu explicité. Faut-il interpréter ce phénomène comme la manifestation d'une indifférence face à une réalité trop lointaine - et parce que finalement Les Mystères de Paris auraient déjà constitué une réalité un peu " exotique " pour le lectorat tchèque? Ou d'une incompréhension face aux " raffinements » des classifications anthropologiques, jugés peu à leur place dans une œuvre de divertissement ? Ou encore d'une réticence face à l'excès incarné par Cecily ? Ces hypothèses, développées à partir de l’examen d'un corpus restreint, demanderaient à être développées à une plus large échelle, en examinant notamment d’autres traductions du XIXe siècle, dans les Pays tchèques, d’œuvres mettant en jeu des problématiques similaires. On peut ainsi penser aux succès populaires de Fenimore Cooper (auteur cité, comme on l'a vu, dans l'incipit des Mystères de Paris) ou de Karl May (extrêmement diffusé dans les dernières décennies du siècle), au best-seller La Case de l'oncle Tom (1852), ou encore au roman Ourika de Claire de Duras (1823), traduit en tchèque en 1863. Les traductions de Bug-Jargal de Hugo (1826) ou Atar-Gull (1831) de Sue, par exemple, apporteraient sans doute des éléments de comparaison utiles, mais plutôt dans une perspective diachronique dans la mesure où elles ne datent respectivement que de 1924 et de $1927^{31}$. Il conviendrait de prendre un compte un corpus transnational, génériquement très varié, incluant sources scientifiques, paralittéraires, lexicographiques et iconographiques, afin de dégager des résultats plus larges sur la circulation des imaginaires racialistes dans une Europe toujours plus confrontée à la découverte d'autres cultures, mais selon des modalités et des degrés bien variables selon les régions.

\section{Références bibliographiques}

\section{Corpus primaire}

Sue, E. (2009). Les Mystères de Paris. Paris : Quarto Gallimard.

(1830). Lettres sur la Guadeloupe n. I, II, III. Revue des Deux Mondes, IV, octobre-novembre

1830, 12-20 ; Lettres sur la Guadeloupe n. IV, V. Revue des Deux Mondes, IV, décembre 1830, 331-347.

. (1862). Tajnosti pařižské : román sociální. Praha : H. Silber (traducteur inconnu).

. (1924). Tajnosti pařižské. Praha-Žižkov : Naklad. Borský a Šulc ${ }^{32}$.

. (1929). Tajnosti pařižské. Přeložil a upravil Karel Čvančara, ilustroval O. Ušák. Praha : Rodinná

knihovna.

. (1970). Tajnosti pařǐžské. Přeložila a upravila Věra Dvořáková. Praha : Odeon.

. (1843). Pariser Mysterien. Ein Sittengemälde aus der neuesten Zeit. Deutsch bearbeitet von Erwin

von Moosthal. Stuttgart : Verlag der Frank'schen Buchhandlung.

. (1844). Die Geheimnisse von Paris. Deutsch von Dr August Diezmann. Leipzig : Wigand.

31 Il faudrait pourtant ne pas oublier l'adaptation raccourcie de Dalibor Kopecký. $C f$. Buk-Jargal. Pověst po Viktoru Hugo in Pověsti z nové romantiky (1839).

32 Traduit par Pr F. Potměšil selon Fryčer (2008 : 94). 


\section{Corpus élargi (XIX ${ }^{\mathrm{e}}$ siècle)}

Archives de Př́ruční slovník jazyka českého (PSJČ) https://psjc.ujc.cas.cz/.

Boiste, P. C. V. (1828). Dictionnaire universel de la langue française avec le latin et les étymologies. Bruxelles: Frechet.

Bescherelle, L.-N. (1856). Dictionnaire national ou Dictionnaire universel de la langue française. Paris : Garnier frères.

Conversations-Lexikon. In fünfzehn Bänden (1843). Leipzig : F. A. Brockhaus.

Conversations-Lexikon der neuesten Zeit und Literatur. In vier Bänden (1832). Leipzig : F. A. Brockhaus.

Dictionnaire de l'académie française (1835). Paris : Imprimerie et librairie de Firmin Didot frères.

Hugo, V. (1888). Bug-Jargal. Paris : E. Hugues (consultable sur Gallica).

Klouček, J. ; \& Žižka, L. K. (1889). Avant-Propos. La langue française en Bohême. In Přehled překladi̊ z jazyka francouzského do češtiny (pp. IX-XXII). Praha : Alliance française.

La Châtre, M. (1852). Dictionnaire universel, Panthéon historique, littéraire et encyclopédie illustrée. Paris : Administration de librairie.

Landais, N. (1852). Dictionnaire classique de la langue française. Paris : Didier.

Laveaux, J.-Ch. (1820). Le Nouveau dictionnaire de la langue française. Paris : Deterville.

Moreau de Saint Méry, L.-E. (1875). Description topographique, physique, civile, politique et historique de la partie française de l'Île Saint-Domingue. Paris : L. Guérin et T. Morgand.

Neruda, J. (1957). Škodlivé směry. In Literatura I (pp. 90-99). Praha : Státní nakladatelství krásné literatury, hudby a umění.

Ottưv slovník naučný (1888-1909). Praha : J. Otto.

Raymond, F. (1832). Dictionnaire général de la langue française et vocabulaire universel des sciences, des arts et des métiers. Paris : A. André, Crochard, F. G. Levrault.

Sabina, K. (1858). Slovo o románu vůbec a o českém zvláště. Lumír, 8, 1-50, 16-1193.

Slovník naučný (1860-1874). Praha : I. L. Kober.

Sobotka, P. (1877). O překladech v novočeské literatuře. Osvěta, 7, 304-320.

\section{Ouvrages critiques}

Blanchard, P. et al. (2011). Zoos humains et exhibitions coloniales. 150 ans d'invention de l'Autre. Paris : La Découverte.

. (2018). Sexe, race et colonie. La domination des corps du XVe siècle à nos jours. Paris : La Découverte.

Bory, J-L. (2000). Eugène Sue. Paris : Mémoire du Livre.

Bourse, A. (2017). Le Métis, une identité hybride? Paris : Classiques Garnier.

Duchet, C. (1968). L'image de Sade à l'époque romantique. In Le marquis de Sade. Paris : A. Colin.

Fryčer, J. (2008). Eugène Sue en Bohême et en Moravie. Le Rocambole. Eugène Sue à l'étranger, 42, 81-95.

Grubitzsch, H. (1977). Materialen zur Kritik des Feuilleton-Romans Die Geheimnisse von Paris von Sue. Wiesbaden : Athenaion.

Merhaut, L. et al. (2008). Lexikon české literatury. Praha : Academia.

Nathan, M. (1990). Splendeurs et misères du roman populaire. Lyon : Presses Universitaires de Lyon.

Poučová, M. (2020). Les traductions tchèques du roman populaire français au XIXe et au début du XX siècles. Belphégor, 18, 1. http://journals.openedition.org/belphegor/2396 
Régent, F. (2011). Structures familiales et stratégies matrimoniales des libres de couleur en Guadeloupe au XVIII siècle. Annales de démographie historique, 2 (2), 69-98. https://doi.org/10.3917/adh.122.0069

Saada, E. (2007). Les Enfants de la colonie. Les métis de l'Empire français entre sujétion et citoyenneté. Paris : La Découverte.

Sainte-Beuve, C. (1843). Quelques vérités sur la situation en littérature. La Revue des deux mondes, $1^{\mathrm{er}}$ juillet $1843,5-20$.

Svane, B. (1988). Si les riches savaient! Le Monde d'Eugène Sue III. Copenhague : Institut de langue et de littérature.

Thérenty, M.-E. ; \& Kalifa, D. (2016). Introduction. In Les Mystères urbains au XIX siècle : Circulations, transferts, appropriations, Médias 19. http://www.medias19.org/index.php?id=21999 